\title{
Setup for the dynamic calibration of bridge amplifiers from DC up to $10 \mathrm{kHz}$
}

\author{
Leonard Klaus, M. Florian Beug, Thomas Bruns \\ Physikalisch-Technische Bundesanstalt (PTB), Bundesallee 100, 38116 Braunschweig, Germany
}

\section{ABSTRACT}

Measurements of mechanical quantities are often carried out with transducers with a bridge output. The output signals are conditioned using bridge amplifiers. If dynamically changing quantities are going to be measured traceably, the bridge amplifier must be calibrated dynamically.

This paper describes a dynamic bridge amplifier calibration setup based on the new PTB dynamic bridge standard. The calibration is carried out by the synchronous sampling of the bridge amplifier output voltage and a reference signal provided by the calibrated dynamic bridge standard. The dynamic bridge standard enables calibrations in a frequency range from DC (static calibration) up to $10 \mathrm{kHz}$. An overview of the different measurement uncertainty contributions is given, and the first measurement results show good agreement with a previously established measurement setup.

\section{Section: RESEARCH PAPER}

Keywords: dynamic bridge amplifier calibration; traceability; dynamic measurement; dynamic bridge standard

Citation: Leonard Klaus, M. Florian Beug, Thomas Bruns, Setup for the dynamic calibration of bridge amplifiers from DC up to $10 \mathrm{kHz}$, Acta IMEKO, vol. 8 , no. 1, article 4, March 2019, identifier: IMEKO-ACTA-08 (2019)-01-04

Section Editor: Maija Ojanen-Saloranta, VTT Technical Research Centre of Finland, National Metrology Institute MIKES, Finland

Received August 15, 2018; In final form October 10, 2018; Published March 2019

Copyright: @ 2019 IMEKO. This is an open-access article distributed under the terms of the Creative Commons Attribution 3.0 License, which permits unrestricted use, distribution, and reproduction in any medium, provided the original author and source are credited

Corresponding author: Leonard Klaus, leonard.klaus@ptb.de

\section{INTRODUCTION}

Mechanical quantities, such as acceleration, force, torque, and pressure, are often measured with transducers, based on strain measurement by means of strain gauges, or sensor elements, based on the piezoresistive effect. These sensing elements change their resistance proportionally to the strain (strain gauge) or the compression/tension (piezoresistive element). However, both transducer principles require signal conditioning by bridge amplifiers.

In many applications, these mechanical quantities change rapidly over time, thus requiring a dynamic calibration in order to be traceable. Until now, bridge amplifiers - as well as the corresponding transducers - have almost exclusively been calibrated statically.

To overcome this problem, a joint European project [1], [2] researched the necessary procedures, measuring devices, and mathematical tools to establish dynamic calibration. For signalconditioning electronics (including all types of measuring amplifiers), it is known that deviations between static and dynamic behaviour exist [3]. These deviations may influence dynamic measurements significantly. Therefore, one outcome of this project was a newly developed dynamic bridge standard [4] that enables bridge amplifier calibration from a static regime (DC) up to frequencies of $10 \mathrm{kHz}$. This bridge standard is incorporated in the calibration setup described here.

This paper is a revised and extended version of [5].

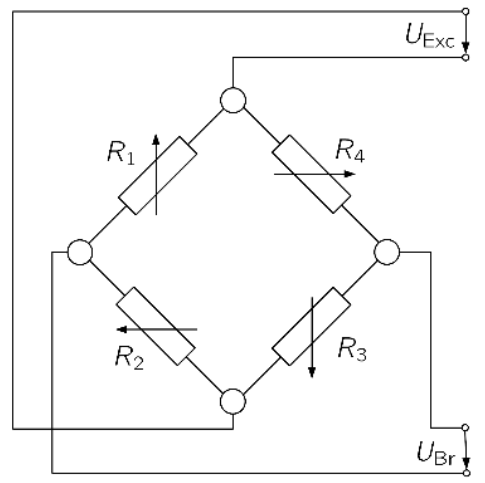

Figure 1: Wheatstone bridge circuit with four resistive sensing elements $R_{1} \ldots R_{4}$, excitation voltage $U_{\mathrm{Exc}}$, and bridge output voltage $U_{\mathrm{Br}}$. 


\section{DYNAMIC BRIDGE STANDARD}

Bridge amplifiers are always connected to some type of Wheatstone bridge circuit, as depicted in Figure 1. The resistive sensing elements (e.g. the strain gauges or piezoresistive sensor elements) are connected as a quarter bridge (one sensing element of variable resistance), half bridge (two sensing elements), or full bridge (four sensing elements) in order to maximise the output signal of the bridge. What all configurations of a Wheatstone bridge have in common is that the output voltage level $U_{\mathrm{Br}}$ depends on the bridge excitation voltage $U_{\mathrm{Exc}}$ (in this case a DC voltage) featuring a ratiometric output, which is typically given in $\mathrm{mV} / \mathrm{V}$. The excitation voltage is supplied by the bridge amplifier. The output of the bridge amplifier is therefore proportional to the ratio of the bridge output voltage to the excitation voltage.

As a result, the bridge output voltage $U_{\mathrm{Br}}$ has no connection to ground. In the bridge amplifier calibration, this bridge voltage $U_{\mathrm{Br}}$ must be ratiometrically provided by the dynamic bridge standard based on the excitation voltage, undertaken in the form of a calibrated voltage ratio and phase.

The dynamic bridge standard working principle is based on two multiplying digital-to-analogue converters (MDACs), which generate output voltages proportional to their reference voltage without dependency to ground. This reference voltage is chosen as the bridge excitation voltage $\pm U_{\mathrm{Exc}}$. A schematic diagram of the dynamic bridge standard components can be seen in Figure 2. Subsequent to the MDAC outputs, a resistive $1 / 400$ voltage divider supplies the small output voltages as required for the bridge amplifier calibration. The load of a strain gauge transducer is simulated by a load resistance of $350 \Omega$. The output voltage of the two MDACs is used as reference for the phase calibration. For this purpose, it is conditioned by buffer amplifiers to avoid any influence on the MDAC output and is fed to connectors placed on the front panel.

The waveforms generated by the MDACs, which can be either static or time-dependent (arbitrary or sinusoidal), are programmed by using an optical computer link.

\section{IMPLEMENTATION OF A CALIBRATION SETUP}

For the calibration of a bridge amplifier, not only is a bridge standard required, but additional data acquisition hardware, a proper data analysis, and a measurement uncertainty evaluation are also required.

The calibration setup incorporates the dynamic bridge standard described as well as a data acquisition system featuring two synchronised sampled data channels. The connection of the different components, including the device under test (DUT), is depicted in Figure 3.

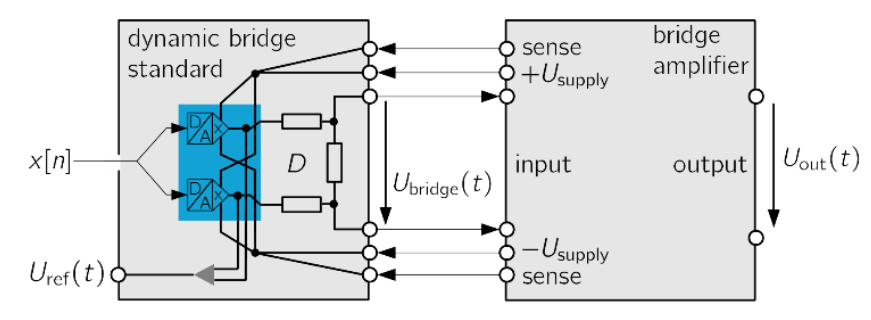

Figure 2. Schematic overview of the dynamic bridge standard.

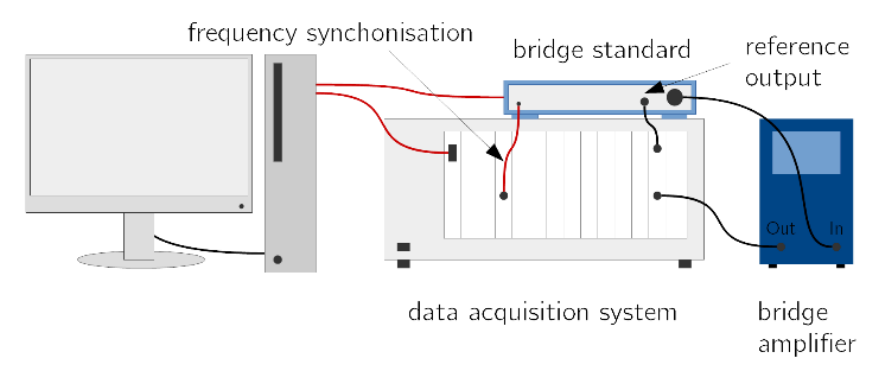

Figure 3. Components of the dynamic bridge amplifier calibration setup.

The dynamic behaviour of an amplifier can be described by its frequency dependent complex transfer function $\underline{H}(\mathrm{i} \omega)$, with its input $\underline{X}(\mathrm{i} \omega)$ and its output $\underline{Y}(\mathrm{i} \omega)$ giving

$\underline{H}(\mathrm{i} \omega)=\frac{Y}{\underline{X}(\mathrm{i} \omega)}$.

More commonly, it is given as a magnitude response $A(\omega)$ and phase response $\varphi(\omega)$, giving

$A(\omega)=\frac{A_{Y}(\omega)}{A_{X}(\omega)}$,

$A \varphi(\omega)=\varphi_{Y}(\omega)-\varphi_{X}(\omega)$,

with the magnitudes $A_{X}, A_{Y}$, and phase angles $\varphi_{X}, \varphi_{Y}$ of the input and output. Based on the magnitude and phase responses, the associated complex transfer function can be derived and vice versa [3].

In the case of the calibration of a bridge amplifier, the amplifier's magnitude excitation at the input $A_{X}$ is well known, because the dynamic bridge standard is calibrated. For the determination of the magnitude response, only the output of the amplifier $A_{Y}$ needs to be analysed. This could be done by means of a calibrated sampling system or even a calibrated AC voltmeter. The phase response determination requires the phase measurement between the output of the bridge amplifier $\varphi_{\mathrm{Y}}(\omega)$ and the dynamic bridge standard's reference signal, since this signal has a calibrated phase relation to the input of the bridge amplifier $\varphi_{\mathrm{X}}(\omega)$.

\section{DATA ACQUISITION}

In our setup (Figure 4), we simultaneously sample the bridge amplifier output and the dynamic bridge standard reference signal by means of two synchronous sampling data acquisition channels. The hardware used for the data acquisition is a PXIe system with a high-resolution digitiser card from National Instruments (PXI-59221), which has a flexible resolution of 18 bits to 24 bits. With sampling rates applicable to the calibration of bridge amplifiers $\left(f_{\mathrm{s}} \ll 500 \mathrm{kHz}\right)$, the resolution is 24 bits. The PXI-5922 digitiser card was thoroughly analysed in terms of its dynamic properties, precision, and metrological suitability [6], [7]. Additionally, automated calibration procedures exist for this acquisition card at PTB (Section 6), as it is widely used in the Realization of Acceleration working group.

The oscillators of the different systems, namely the oscillator of the dynamic bridge standard and of the data acquisition system, are synchronised in order to avoid spectral leakage. The dynamic bridge standard is equipped with an optical clock input. The PXIe system is equipped with a timing and synchronisation

\footnotetext{
${ }^{1}$ Commercial instruments are identified in this paper only to adequately specify the experimental setup. Such identification does not imply recommendation by PTB, nor does it imply that the equipment identified is necessarily the best available for the purpose.
} 


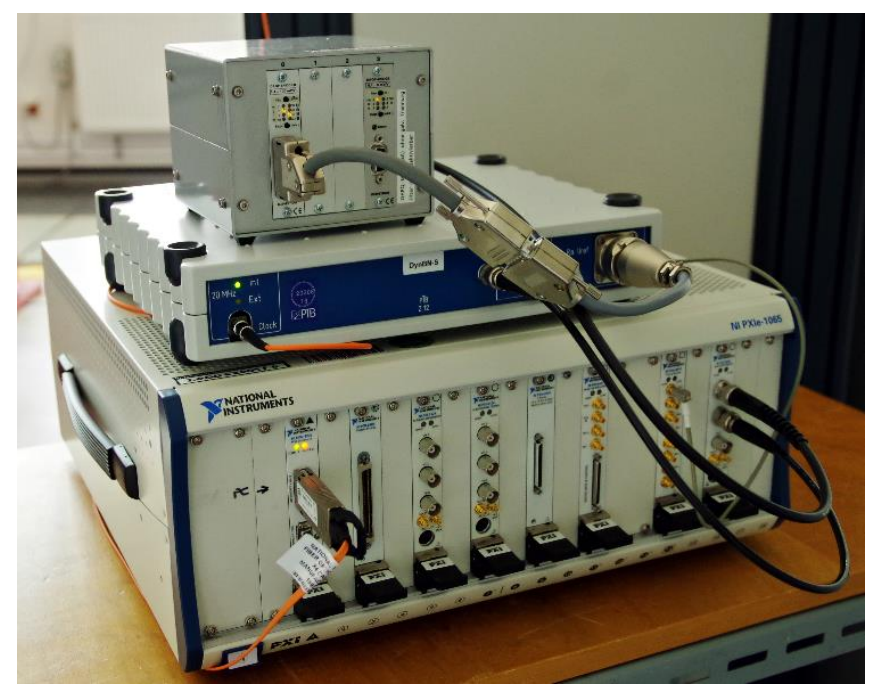

Figure 4. Dynamic bridge amplifier calibration setup, consisting of the bridge amplifier under test (top), the dynamic bridge standard (middle), and the data acquisition system (bottom).

card (PXIe-6672), which features a stable oscillator (TCX0). Its one-year stability is in the range of a few $10^{6}$ [8]. The $20 \mathrm{MHz}$ reference frequency for the dynamic bridge standard is then generated by direct digital synthesis (DDS) based on the $10 \mathrm{MHz}$ TCX0 oscillator. The electrical frequency output provided by the PXIe card is converted to an optical link as required by the bridge standard.

Dynamic bridge excitation signals are generated by two MDACs with a sampling frequency that is a fraction of the reference frequency. Based on this sampling frequency, the sinusoidal waveforms can be generated based on a finite number of data points per oscillation period, which are then repeatedly output. The sampling frequencies and the acquisition time of the data acquisition were chosen based on two criteria:

1) The sampling rate should be multiples or integer fractions of the bridge standard's sampling rate. Generally, it would not be favourable to sample with a higher sampling rate than the signal generation rate. However, the chosen digitiser card has a minimum sampling rate of $50 \mathrm{kS} / \mathrm{s}$, requiring a down-sampling of the data after acquisition. This decimation process is carried out by calculating an averaged decimated waveform from the original data.

2) The sampling rate was chosen in order for us to achieve a finite number of samples for the selected number of oscillations requiring acquisition. The corresponding acquisition time should include an integer number of periods of the excitation frequency.

The sampling frequency of the chosen digitiser cannot be chosen arbitrarily. It is chosen as an even integer divider of the sampling frequency of $120 \mathrm{MS} / \mathrm{s}$ for the bitstream input of the incorporated delta-sigma analogue-to-digital converters (ADCs) and must be in a range of $50 \mathrm{kS} / \mathrm{s}$ and $15 \mathrm{MS} / \mathrm{s}$.

\section{DATA ANALYSIS}

The acquired and synchronously sampled waveforms of the two input channels make up the input for the data analysis. One data channel is the reference output of the dynamic bridge standard, and therefore contains a signal with a known relation to the input of the transducer, while the second data channel contains the voltage output of the device under test.
The transformation of the time series data into the frequency domain is carried out by means of digital Fourier transform (DFT). Because of the synchronised generation and acquisition frequencies, spectral leakage can be avoided. The sampling of full periods of the sinusoidal waveform allows us to go without any windowing prior to the DFT.

Based on the outcomes of the two DFTs and the calibration results of the dynamic bridge standard, the magnitude and phase responses of the DUT at each excitation frequency can be calculated as described in Section 3. The magnitude response is derived only based on the output of the device under test, as the input magnitude is known from the bridge standard's calibration data. The phase response (i.e. the frequency-dependent phase delay) requires input from both measurement channels, as the absolute phase position cannot be derived from calibration data.

For each frequency point, repeated measurements are carried out (the number of repetitions can be set), and the mean and the standard deviation are calculated, giving information about the stability of the input-output relation of the DUT for each calibration frequency.

\section{MEASUREMENT UNCERTAINTY EVALUATION}

The measurement uncertainty is evaluated according to the Guide to the Expression of Uncertainty in Measurement (GUM) [9] and Supplement 1 thereto (GUM S1) [10] by means of Monte Carlo simulations.

GUM distinguishes between two types of measurement uncertainty contributions, both of which are considered for the measurement uncertainty estimation:

1) Type A uncertainty contributions: These can be determined by the statistical analysis of measurements.

2) Type B uncertainty contributions: These include all contributions that cannot be estimated by statistical analysis. They may include datasheet content, results from calibrations (of, for example, components of the setup), operator know-how, records from previous measurements, or other prior knowledge.

The measurement is modelled by means of mathematical model function $f$ propagating all the input quantities $X_{1} \ldots X_{n}$ to the measurement result $Y$, giving

$Y=f\left(X_{1}, X_{2}, \ldots, X_{n}\right)$.

Each input quantity can be described mathematically by its distribution, which leads to estimations of the value of the input quantity and its uncertainty. Depending on the sources of information about the input quantity and its properties, the corresponding distributions (Gaussian distributed, rectangularly distributed, etc.) are assigned.

Independent calibrations of the components of the calibration setups are incorporated in the uncertainty estimation as sub-models, i.e. the distributions of the calibration results are assigned to the according input quantity $X$. This is the case for the two components detailed below.

\subsection{Calibration of the dynamic bridge standard}

The bridge standard was calibrated in advance by calibrating the resistive voltage divider, the MDACs, and the signal conditioners of the outputs. The expanded measurement uncertainties $(U(k=2))$ of this calibration are $0.03 \%$ (magnitude) and $0.05^{\circ}$ (phase angle) for frequencies up to $1 \mathrm{kHz}$ and $0.05 \%$ (magnitude) and $0.1^{\circ}$ (phase angle) for frequencies above $1 \mathrm{kHz}$ and up to $10 \mathrm{kHz}$. 

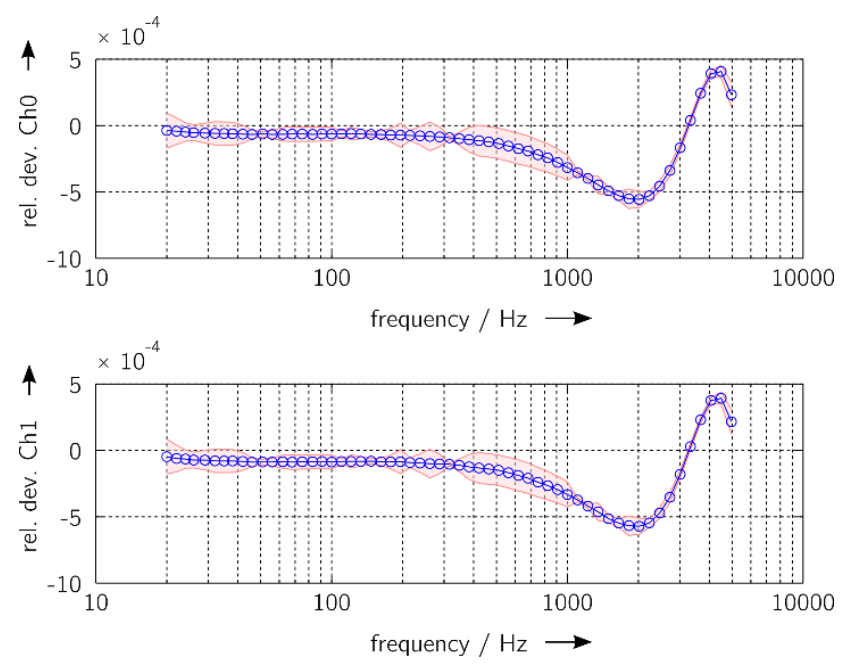

Figure 5: Calibration results for a PXI-5922 digitiser card. The relative deviations are given in blue, and the assigned measurement uncertainty $U(k=2)$ is red. The sampling rate was $50 \mathrm{kS} / \mathrm{s}$, and the input voltage span $\pm 5 \mathrm{~V}$.

There are systematic effects, which are in the same order as the expanded measurement uncertainty for the magnitude transfer function and less than $0.2^{\circ}$ for the phase angle at $10 \mathrm{kHz}$.

\subsection{Calibration of the digitiser channels}

The two digitiser input channels were calibrated dynamically using a voltage calibrator. In typical conditioning amplifier calibration setups, it is not necessary to calibrate the data acquisition hardware so thoroughly if the input and output are acquired simultaneously and a second measurement with swapped input channels is carried out. In this case, possible influences due to the different input channel characteristics can be corrected. However, in the case of the dynamic bridge standard, this procedure is not applicable: As the magnitude response is only derived from the bridge standards output (Section 3), all influences due to the digitalisation need to be determined.

The calibration of the digitiser channels was carried out using a Fluke 5700A voltage calibrator (which was itself calibrated dynamically beforehand). For the calibration, both input channels were connected to the voltage calibrator's outputs, and the voltages of known magnitude and frequency were generated. From the acquired signal, the root mean square (RMS) value was derived for each frequency point of the calibration. The digitiser card shows a characteristic transfer function, which could be compensated for as it is only dependent on the sampling rate [6],

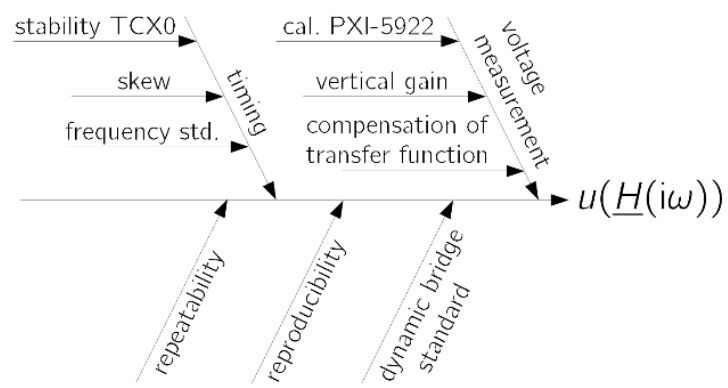

Figure 6. Ishikawa diagram of the measurement uncertainty contributions for a dynamic bridge amplifier calibration.

[7]. Figure 5 shows the calibration results for a sampling rate of $50 \mathrm{kS} / \mathrm{s}$ (the same rate used for the calibration measurements).

The stable local oscillator of the PXIe timing and synchronisation card was calibrated traceably to PTB's frequency standards by means of a distributed frequency signal.

The different measurement uncertainty contributions are depicted in Figure 6, and detailed information about the different type $\mathrm{B}$ contributions, including their distributions, is given in Table 1.

\subsection{Statistical analysis of the measurements}

The type A uncertainty contributions are also analysed. The stability of the measurement setup and of the different DUTs are assessed by carrying out many repeated measurements in a short time (referred to as 'repeatability' in Figure 6) and by repeating the calibration at larger intervals, including the disconnection of all cables and turning the calibration devices and devices under test off between measurements (referred to as 'reproducibility').

A complete evaluation of the type A uncertainty contributions requires extensive measurements with different DUTs and numerous repetitions, which have not yet been carried out.

\subsection{Correction of systematic effects}

Both the signal acquisition card and the dynamic bridge standard show a frequency-dependent change in their transfer function. GUM recommends a correction of all systematic effects. However, if necessary, it is possible to treat those systematic effects as uncertainty contributions [11]. Both the magnitude-related systematic contributions are in the same range as the random measurement uncertainty component of the calibration of the dynamic bridge standard. Therefore, in the first step, these are not corrected; rather, they are included in the measurement uncertainty estimation as input quantities. In future, these could be corrected to reduce the resulting measurement uncertainty for the bridge amplifier calibration.

Table 1. Type B measurement uncertainty contributions of the calibration setup.

\begin{tabular}{|c|c|c|}
\hline Input quantity & Uncertainty contribution & Distribution \\
\hline Dynamic bridge standard (magnitude) & $u_{\text {rel }} \leq 2.5 \times 10^{-4}$ & Gaussian \\
\hline Dynamic bridge standard (phase) & $u \leq 0.05^{\circ}$ & Gaussian \\
\hline Dynamic bridge standard systematic (mag.) & $u_{\mathrm{rel}} \leq 5 \times 10^{-4}$ & Gaussian \\
\hline Frequency standard & $u_{\mathrm{rel}}=1 \times 10^{-10}$ & Gaussian \\
\hline Stability TCXO (1 year) & $u_{\text {rel }}=1 \times 10^{-6}$ & rectangular \\
\hline Temperature stab. TCXO $\left(0{ }^{\circ} \mathrm{C}-55^{\circ} \mathrm{C}\right)$ & $u_{\mathrm{rel}}=2 \times 10^{-6}$ & rectangular \\
\hline Skew & $u=5 \times 10^{-10} \mathrm{~s}$ & rectangular \\
\hline Cal. PXI-5922 (magnitude) & $u_{\text {rel }} \leq 2.5 \times 10^{-6}$ & Gaussian \\
\hline PXI-5922 systematic deviations (magnitude) & $u_{\mathrm{rel}}=6 \times 10^{-4}$ & Gaussian \\
\hline PXI-5922 DC accuracy (magnitude) & $\begin{aligned} u_{\text {rel }} & =5 \times 10^{-4} \text { and } \\
u & =1 \times 10^{-5} \mathrm{~V}\end{aligned}$ & $\begin{array}{l}\text { rectangular } \\
\text { rectangular }\end{array}$ \\
\hline
\end{tabular}




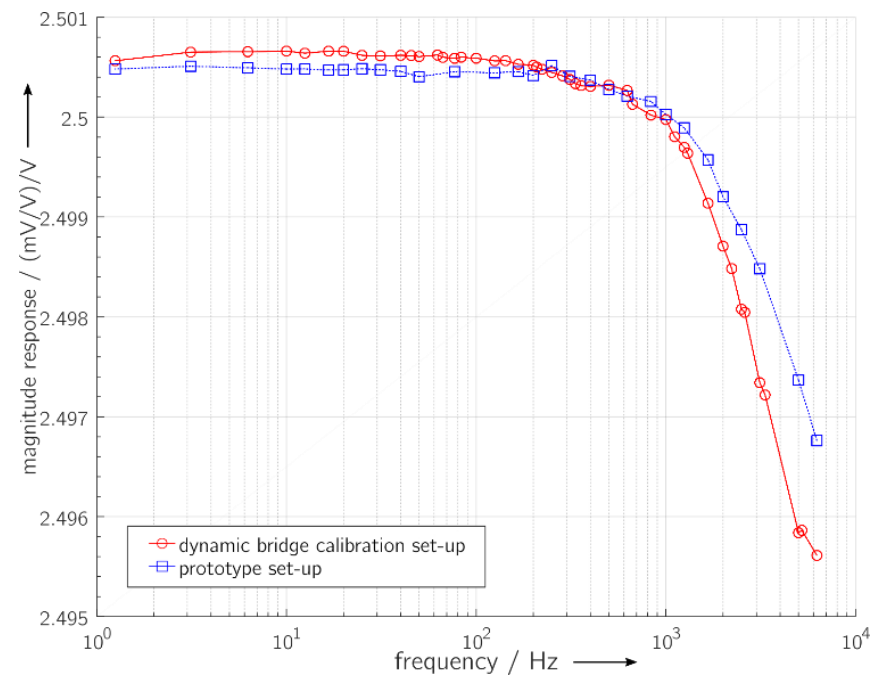

Figure 7. Magnitude response calibration results of a bridge amplifier using a prototype of the bridge standard (blue) and of the actual setup (red).

\subsection{Application of the Monte Carlo simulation}

The measurement uncertainty evaluation is carried out by means of a Monte Carlo simulation according to GUM S1. For this purpose, repeated simulations of the measurement are carried out. The different input quantities are modelled according to their input distributions. The resultant distribution of the output describes the measurement results, including uncertainty.

\section{FIRST MEASUREMENT RESULTS}

To obtain a first impression of the performance of this new setup, measurements are carried out with a bridge amplifier under test, which had previously been dynamically calibrated. The previous calibration had been carried out with an earlier prototype of the dynamic bridge standard in conjunction with a sampling voltmeter [12]. The results of the magnitude and phase responses are given in Figure 7 and Figure 8, respectively.

The chosen bridge amplifier shows only small deviations (in the range of a few $10^{-3}$ for the magnitude response) from the ideal behaviour in the chosen frequency range. The measurement

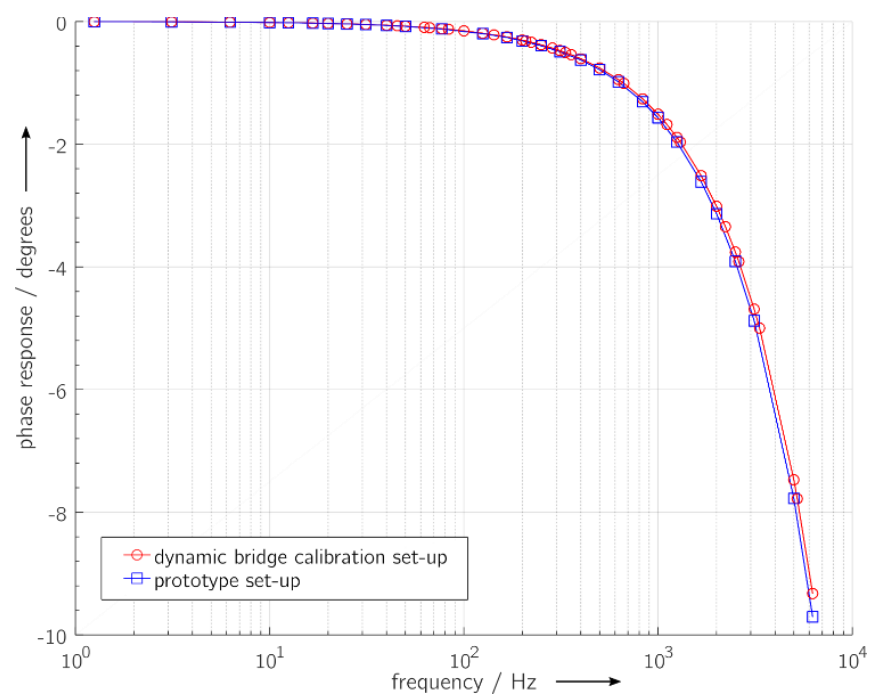

Figure 8: Phase response calibration results of a bridge amplifier using a prototype of the dynamic bridge standard (blue) and of the actual setup (red). results agree very well both in terms of magnitude and phase, despite that the individual frequency responses of the corresponding bridge standards have not yet been compensated for. The differences between the results of the two measurements are even slightly smaller than the largest single type B measurement uncertainty contribution.

\section{CONCLUSION}

This paper describes a dynamic bridge amplifier calibration setup based on the new PTB dynamic bridge standard. The output of the bridge standard and the DUT is sampled synchronously by a high-precision digitiser. A synchronisation of the oscillators of both the data acquisition system and the bridge standard avoids spectral leakage.

The new calibration setup enables the dynamic calibration of bridge amplifiers according to the measurement conditions required by ISO 4965-2 [13], in which a dynamic signal on a constant bias is stipulated.

The first measurement results of the new setup agree very well with measurements previously carried out with a prototype of the dynamic bridge standard.

\section{REFERENCES}

[1] C. Bartoli et al., Traceable dynamic measurement of mechanical quantities: Objectives and first results of this European project, International Journal of Metrology and Quality Engineering 3, 3 (2013) pp. 127-135,

DOI: $10.1051 /$ ijmqe $/ 2012020$.

[2] C. Bartoli et al., Dynamic calibration of force, torque, and pressure sensors, in Proc. of the Joint IMEKO International TC3, TC5 and TC22 Conference 2014, Cape Town, South Africa, 2014, https://www.imeko.org/publications/tc22-2014/IMEKO-TC3TC22-2014-007.pdf.

[3] L. Klaus, T. Bruns, H. Volkers, Calibration of bridge-, charge-, and voltage amplifiers for dynamic measurement applications, Metrologia 52, 1 (2015) pp. 72-81, DOI: $10.1088 / 0026-1394 / 52 / 1 / 72$.

[4] M. F. Beug, H. Moser, G. Ramm, Dynamic bridge standard for strain gauge bridge amplifier calibration, in Proc. of the 2012 Conference on Precision Electromagnetic Measurements (CPEM), 2012, Washington DC, USA, pp. 568-69, DOI: 10.1109/CPEM.2012.6251056.

[5] L. Klaus, M.F. Beug, T. Bruns, A new calibration set-up for the dynamic calibration of bridge amplifiers from DC up to $10 \mathrm{kHz}$, in Proc. of the $23^{\text {rd }}$ TC $3,13^{\text {th }}$ TC5 and $4^{\text {th }}$ TC22 IMEKO International Conference, Helsinki, Finland, 2017, https://www.imeko.org/publications/tc3-2017/IMEKO-TC32017-011.pdf.

[6] G. Rietvield et al., Characterization of a wideband digitizer for power measurements up to $1 \mathrm{MHz}$, IEEE Transactions on Instrumentation and Measurement 60, 7 (2011) pp. 2195-2201, DOI: $\underline{10.1109 / T I M .2011 .2117330 . ~}$

[7] F. Overney et al., Characterization of metrological grade analogto-digital converters using a programmable Josephson voltage standard, IEEE Transactions on Instrumentation and Measurement 60, 7 (2011) pp. 2172-2177,

DOI: 10.1109/TIM.2011.2113950.

[8] National Instruments Corporation, NI PXIe-6672 User Manual, 2010.

[9] Bureau International des Poids et Mesures, et al., Evaluation of Measurement Data - Guide to the Expression of Uncertainty in Measurement, 2008.

[10] Bureau International des Poids et Mesures, et al., Evaluation of Measurement Data - Supplement 1 to the Guide to the Expression of Uncertainty in Measurement - Propagation of Distributions using a Monte Carlo Method, 2008. 
[11] I. H. Lira, W. Wöger, Evaluation of the uncertainty associated with a measurement result not corrected for systematic effects, in Measurement Science and Technology 9(6) (1998) p. 1010, DOI: $10.1088 / 0957-0233 / 9 / 6 / 019$.

[12] L. Klaus, Entwicklung eines primären Verfahrens zur Kalibrierung von Drehmomentaufnehmern mit dynamischer Anregung, PTB
Report MA-93, 2016, pp. 59-61, pp. 146-50, DOI: $10.7795 / 110.20160714$.

[13] ISO/TC 164/SC 5, ISO 4965-2:2012, Metallic Materials Dynamic Force Calibration for Uniaxial Fatigue Testing - Part 2: Dynamic Calibration Device (DCD) Instrumentation, International Organization for Standardization, 2012. 